\title{
The cleaning effect of sugar-free chewing gum on occlusal surfaces
}

\author{
Harpreet Kaur Sindhu*, Sri Wendari Hartono*, Endang Sukartini** \\ Department of Periodontics Faculty of Dentistry Universitas Padjadjaran \\ **Department of Conservative Dentistry Faculty of Dentistry Universitas Padjadjaran
}

\begin{abstract}
Introduction: Sugar-free chewing gum has the potential to provide oral health benefits including plaque control. This study benefit to develop and validate an occlusal site-specific plaque index. The index will be used to check the results of chewing sugar-free chewing gum. The purpose of this study is to analized the cleaning effect of sugar-free chewing gum on occlusal surfaces. Methods: The research is a split mouth design, single blind, cross over study. The research consists of 30 volunteers chosen by random sampling. Volunteers divided into two groups, each consisting of 15 volunteer's. Group A, volunteers were asked to chewing gum on the left side regimen whereas volunteers in group $B$ were asked to chew on the right side regimen for 5 minute period. After a minimum of 4 days washout period, the study was repeated with the volunteers' crossing over to the alternative chewing right or chewing left regimen. Results: The mean difference 0.224 . Paired sample t-test was used to determine whether there are significant differences before and after chewing gum. With level of significance $a=5 \%$ the $t$-values for both groups are both 0.00 . There are differences before and after chewing gum in group $A$ and $B$. Conclusion: Sugar-free chewing gum have cleaning effect of on occlusal surfaces, sugar-free chewing gum decreases the plaque accumulation on occlusal surfaces.
\end{abstract}

Key words: Plaque, sugar-free chewing gum.

\section{INTRODUCTION}

Physical health is an important aspect in the community and starts from self-maintenance. One of the efforts of health maintenance is by maintaining oral health. Ministry of Health reported that more than 70 percent of the population of Indonesia is experiencing caries or cavities and periodontal diseases. ${ }^{1}$

Dental caries is caused by bacterial processes that damage hard tooth structure. Bacteria feed on sugar in the mouth and secrete acid, which dissolves the enamel. Excessive exposure to sugar with poor oral hygiene, allows for an increase in bacterial activity on the tooth, which causes structural damage in the enamel. If left untreated, the caries can penetrate through the dentin layer causing extensive tooth decay. ${ }^{2}$

Dental plaque (oral biofilm) formation is a dynamic and complex process involving many 
stages, from the adsorption of salivary pellicle to bacterial accumulation and growth. More than 700 bacterial species that inhabit the oral cavity participate in this process. Dental caries and periodontal diseases caused by dental plaque, formed as a result of the complex interactions between teeth and adsorbed host and bacterial molecules, passive transport of oral bacteria, co-adhesion of successive bacterial strains, and the multiplication of associated microorganisms. Dental caries are initiated by bacterial adhesion and subsequent plaque formation on the tooth surface, followed by bacterial carbohydrate fermentation and organic acid formation. These acids diffuse into and ultimately demineralize the tooth. For periodontal diseases, the presence of pathogenic plaque, together with host-related factors that modify the response to plaque bacteria, is the key determinant contributing to the development of the diseases. ${ }^{3}$

Dental plaque control can be divided into three, namely mechanical, chemical and combination of mechanical and chemical. ${ }^{4}$ Tooth brushing is a mechanical control of dental plaque and considered as an effective way to inhibit the formation of dental plaque, preventing the occurrence of inflammation of the gums, but in reality it is difficult to completely remove all plaque. ${ }^{5}$ Inability of disposing plaque perfectly reattributes promote researches leading like to use chemical medicines in the form of mouthwash, tooth paste, chewing gum and candy suction. Mouthwash used for cleaning the mouth of food debris. Mouthwash contained antibacterial function to prevent or reduce plaque accumulation. ${ }^{6}$ Mouthwashes provide therapeutic effects that can reduce plaque formation which can prevent gingivitis and also can give a good effect on the inhibition of the formation of caries by adding fluoride to it.

Chewing gum and brushing teeth to control plaque accumulation with combine method such as mechanical and chemical. Chewing gum is one of the means used as a cleaning plaque. Chewing gum is not a modern habit, people have chewed gum-like substances for many centuries. One of the earliest people to 'chew' were the ancient Greeks. They extracted a resin (a sticky solid) from trees. This resin was called mastic gum. The native American-Indians chewed the resin from spruce trees. People in ancient time have probably chewed gum in order to freshen their breath because they would not have had toothpaste. But recent research into chewing sugar free gum can help in the fight against tooth decay. ${ }^{7}$ The mechanical cleaning effects of chewing gums have been studied, usually on established plaque deposits or food debris with equivocal results..$^{8,9}$

Most studies evacuated effects on buccal and lingual plaque deposits presumably where the interest was primarily with potential gingival health benefits of chewing gum. Occlusal plaque indicators have been developed and one of two centre study investigated the effects of chewing gum on established occlusal plaque with equivocal results. ${ }^{10-12} \mathrm{~A}$ research was done by Levinkind et al. Concluded that this site-specific index for occlusal plaque was developed and developed and demonstrated to be repeatable within examiner and reproducible between examiners. ${ }^{12}$

The initial health aim was that of substitution of sugar, in gums mostly sucrose and the usual sugars substitute was sorbitol. Subsequently, the demonstration of the effect of sugar-free gum in raising plaque $\mathrm{pH}$, and the remineralising powers of stimulated saliva, led to these actions becoming additional health gains. The special role of xyllitol is indicated by the fact that not only does it stimulate saliva as do other sweeteners, but in addition it has a well-proved beneficial effect in reducing the cariogenicity of the plaque microflora, and has some interesting potential in the area of demineralization. ${ }^{13}$ The purpose of this study is to analized the cleaning effect of sugarfree chewing gum on occlusal surfaces.

\section{METHODS}

This research was a quasi-experimental, randomized, controlled single blind and split mouth design. The populations for this research are students from SMA PASUNDAN 8, Bandung. 30 volunteers are obtained by randomized sampling. The criteria of the volunteers are as follows: Male or female, 16-17 years of age, not on antibiotic therapy and do not have any systemic disease, do not wear any orthodontic or prosthodontic appliances, no caries, no fillings, have at least 8 gradable occlusal surfaces of premolars and molars, willing to be a subject of research 
(informed consent). Mouth mirror, explorer, tweezers, binocular x 2.4 magnification, glass for rinsing, tissue, face mask and gloves, sugarfree chewing gum (Wrigley's Extra Professional Spearmint), disclosing solution (erythrocin), informed consent, examination sheet.

\section{RESULTS}

The experiment was carried out on 28 subjects, 19 female and 9 male ages between 15 to 16 years old comprising of students of
SMA Pasundan 8, Bandung. Volunteers were divvied into 2 groups, group $A$ and $B$. In group $A$, volunteers were asked to chewing gum on the left side regimen whereas volunteers in group $B$ were asked to chew on the right side. After 4 days washout period, the study was repeated with the volunteers' crossing over to the alternative chewing right or chewing left regimen. Based on those results of research that has been done can be known based on Occlusal Site-specific plaque index between before and after chewing sugarfree chewing gum. It can be seen in table 1, 2 and 3 .

Table 1. The mean of Occlusal Site-specific plaque index group A and B before and after chewing

\begin{tabular}{lccccccc}
\hline & \multirow{n}{*}{$\mathbf{n}$} & \multicolumn{3}{c}{ Day 2 } & \multicolumn{3}{c}{ Day 6 } \\
\cline { 3 - 7 } & & Before & After & Difference & Before & After & Difference \\
\hline Group A & 14 & 0.535 & 0.254 & 0.2897 & 0.423 & 0.241 & 0.1814 \\
Group B & 14 & 0.556 & 0.288 & 0.269 & 0.489 & 0.266 & 0.224 \\
\hline
\end{tabular}

Table 2. The mean of Occlusal Site-specific plaque index group A and B before and after chewing right side and left side before wash out period

\begin{tabular}{cccccccc}
\hline \multirow{2}{*}{ Regimen } & $\mathbf{n}$ & & \multicolumn{3}{c}{ Group A } & & \multicolumn{2}{c}{ Group B } \\
\cline { 3 - 7 } & & Before & After & Difference & Before & After & Difference \\
\hline Left & 14 & 1.025 & 0.264 & 0.761 & 1.051 & 0.901 & 0.150 \\
Right & 14 & 0.997 & 0.723 & 0.273 & 1.112 & 0.253 & 0.859 \\
\hline
\end{tabular}

Table 3. The mean of Occlusal Site-specific plaque index group A and B before and after chewing, right side and left side after wash-out period.

\begin{tabular}{cccccccc}
\hline \multirow{2}{*}{ Regimen } & \multirow{n}{*}{$\mathbf{n}$} & \multicolumn{3}{c}{ Group A } & \multicolumn{3}{c}{ Group B } \\
\cline { 3 - 8 } & & Before & After & Difference & Before & After & Difference \\
\hline Left & 14 & 0.801 & 0.711 & 0.090 & 0.905 & 0.239 & 0.666 \\
Right & 14 & 0.829 & 0.220 & 0.609 & 0.849 & 0.775 & 0.074 \\
\hline
\end{tabular}

Table 4. The mean of Occlusal Site-specific plaque index group A and B before and after chewing

\begin{tabular}{lccccccc}
\hline & \multirow{2}{*}{$\mathrm{n}$} & \multicolumn{3}{c}{ Day 2 } & \multicolumn{3}{c}{ Day 6 } \\
\cline { 3 - 8 } & & Before & After & Difference & Before & After & Difference \\
\hline Group A & 14 & 0.535 & 0.254 & 0.2897 & 0.423 & 0.241 & 0.1814 \\
Group B & 14 & 0.556 & 0.288 & 0.269 & 0.489 & 0.266 & 0.224 \\
\hline
\end{tabular}

Table 5. Percentage mean difference of Occlusal Site-specific plaque index group A and B right and left regimen according to type of tooth

\begin{tabular}{ccccccccc}
\hline \multirow{2}{*}{ Type of tooth } & \multicolumn{3}{c}{ Right } & \multicolumn{2}{c}{ Group A } & \multicolumn{2}{c}{ Group B } \\
\cline { 2 - 9 } & \multicolumn{2}{c}{ Group A } & \multicolumn{2}{c}{ Group B } & Day 2 & Day 6 & Day 2 & Day 6 \\
\cline { 2 - 9 } & $11.67 \%$ & $21.44 \%$ & $34.09 \%$ & $3.90 \%$ & $22.08 \%$ & $9.02 \%$ & $55.10 \%$ & $25.33 \%$ \\
\hline Premolar & $15.68 \%$ & $39.50 \%$ & $51.80 \%$ & $3.53 \%$ & $54.16 \%$ & $9.91 \%$ & $9.48 \%$ & $4.12 \%$ \\
Molar & $15 \%$ &
\end{tabular}




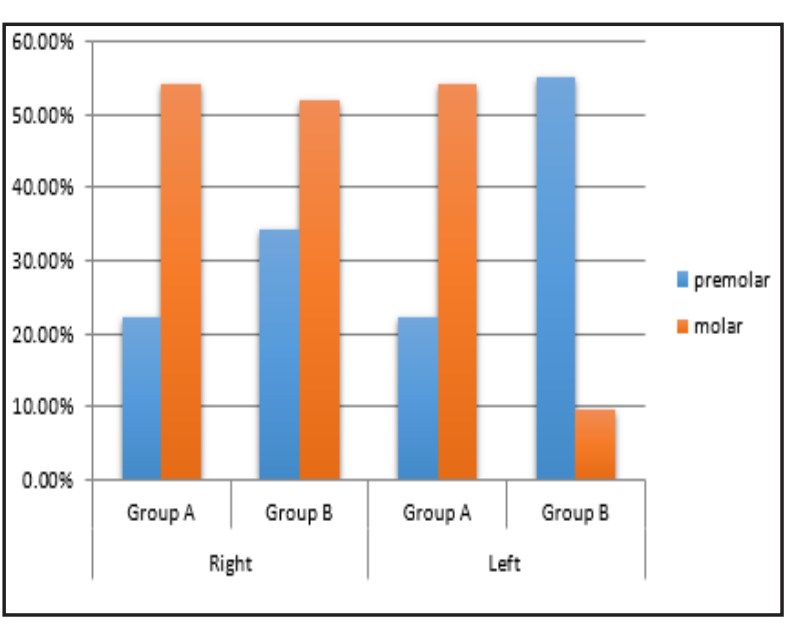

Figure 1. Comparison of mean difference of occlusal site-specific plaque index group A and B on the according to type of tooth before wash-out period

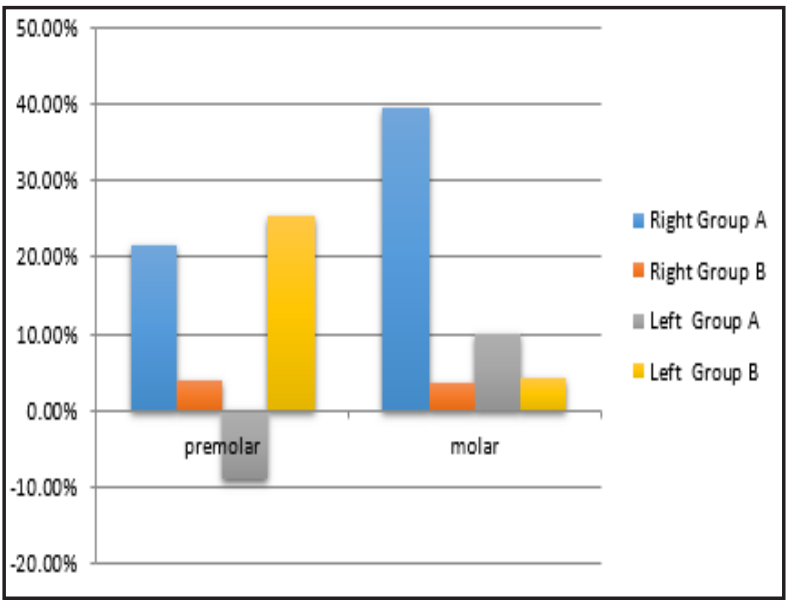

Figure 2. Comparison of mean difference of occlusal site-specific plaque index group $A$ and $B$ according to type of tooth after wash-out period

Based on Table 1 it can be seen that the mean of occlusal site-specific plaque index for group A, day 2 before chewing is 0.535 and after 0.254 . The mean difference is 0.2897 . The mean of Occlusal Site-specific plaque index group A, day 6 before chewing is 0.423 and after 0.241 . The mean difference 0.181 .

The mean of occlusal site-specific plaque index for group B, day 2 before chewing is 0.556 and after 0.288 . The mean difference is 0.269 . The mean of Occlusal Site-specific plaque index group B, day 6 before chewing is 0.489 and after 0.266 . The mean difference 0.224.Paired sample t-test was used to determine whether there are significant differences before and after chewing gum. With level of significance $a=5 \%$ the $\mathrm{t}$-values for both groups are both 0.00 . Therefore there are significant differences before and after chewing gum in group $A$ and $B$.

Based on Table 2 it can be seen that the mean plaque index for before wash- out period regimen left for group A before chewing gum is 1.025 and after is 0.264 . The mean difference is 0.761 . The mean plaque index for before wash-out period regimen right group $\mathrm{A}$ before chewing gum is 0.997 and after is 0.723 . The mean difference is 0.273 .

The mean plaque index for before wash- out period regimen left group $B$ before chewing gum is 1.051 and after is 0.901 . The mean difference is 0.150 . The mean plaque index for before wash-out period regimen right group $B$ before chewing gum is 1.112 and after is 0.253 . The mean difference is 0.859 the highest mean difference of occusal sitespecific plaque.

Based on Table 3 it can be seen that the mean plaque index for after wash- out period regimen left for group $A$ before chewing gum is 0.801 and after is 0.711 . The mean difference is 0.090 . The mean plaque index for after wash-out period regimen right group $A$ before chewing gum is 0.829 and after is 0.220 . The mean difference is 0.609 .

The mean plaque index for after wash- out period regimen left group $B$ before chewing gum is 0.905 and after is 0.239 . The mean difference is 0.666 , the highest mean difference occlusal site-specific plaque index. The mean plaque index for after wash-out period regimen right group $B$ before chewing gum is 0.849 and after is 0.775 . The mean difference is 0.074 .

According to Table 5 and Figure 1 can also be seen that the percentage of mean difference of occlusal site-specific plaque index for group $A$ at right regimen, before wash-out period for premolar is $11.67 \%$ and molar is $15.68 \%$. The percentage of mean difference of occlusal sitespecific index for group $A$, after wash-out period for premolar is $21.44 \%$ and molar is $39.50 \%$.

The percentage of mean difference of occlusal site-specific plaque index for group B at right regimen, before wash-out period for premolar is $34.09 \%$ and molar is $51.80 \%$. The percentage of mean difference of occlusal sitespecific index for group $B$, after wash-out period for premolar is $3.90 \%$ and molar is $3.53 \%$.

According to Table 3 and Figure 2 can also 
be seen that the percentage of mean difference of occlusal site-specific plaque index for group $A$ at left regimen, before wash-out period for premolar is $22.08 \%$ and molar is $54.16 \%$. The percentage of mean difference of occlusal site-specific index for group A, after wash-out period for premolar is $-9.02 \%$ and molar is $9.91 \%$.

The percentage of mean difference of occlusal site-specific plaque index for group $B$ at right regimen, before wash-out period for premolar is $55.10 \%$ and molar is $9.48 \%$. The percentage of mean difference of occlusal site-specific index for group $B$, after wash-out period for premolar is $25.33 \%$ and molar is $4.12 \%$.

\section{DISCUSSION}

Results of research and statistical calculation show that sugar-free chewing gum effectively decreases the value of plaque. This shows that sugar-free chewing gum has the effect of cleaning teeth because it can increase the production of saliva and has a mechanical effect that allows the tooth cleaning effect of food remnants.

A previous study developed an occlusal plaque scoring system based either on a total coverage score not dissimilar from the smooth surface index, or on area, as described for smooth surfaces. ${ }^{11,14,15}$ This index proved repeatable for single examiner and was validated by detecting significant differences in plaque scores after brushing compared to no brushing. For smooth surfaces; site specific indices have developed to increase the sensitivity of indices to detect differences in treatment particularly at designated sites.

During the study, there are no any side effects on research subjects. According to the results of this study, sugar-free chewing gum can reduce plaque accumulation on the occlusal significantly. Although that case, efforts to control plaque by brushing teeth still preferred because it produces mechanical power cleaning more effective plaque. Sugar-free chewing gum can be carried out when brushing your teeth in an effort to maintain oral hygiene support the effectiveness of brushing.

\section{CONCLUSION}

Sugar-free chewing gum have cleaning effect of on occlusal surfaces, sugar-free chewing gum decreases the plaque accumulation on occlusal surfaces.

\section{REFERENCES}

1. World Health Organization. Obesity: preventing and managing the global epidemic. Report of a WHO Consultation (WHO Technical Report Series 894). Geneva: World Health Organization; 2001.

2. Mishra MB, Mishra S. Sugar-Sweetened beverages: General and oral health hazards in children and adolescents. Int J Clin Pediatr Dent. 2011;4(2):119-123.

3. Faraj JA, Dorati R, Schoubben A, Worthen D, Selmin F, Capan Y, et al. Development of a Peptide-containing Chewing Gum as a Sustained Release Antiplaque Antimicrobial Delivery System. AAPS PharmSciTech. 2007 Mar 30;8(1):26.

4. Newman MG, Takei HH, Carranza FA. Carranza's Clinical Periodontology. $10^{\text {th }}$ ed. Philadelphia: Saunders-Elsevier; 2006. p. 137, 728

5. Daliemunthe SH. Obat Kumur dan Kesehatan Periodontium. Majalah Kedokteran Gigi. Jakarta: Universitas Indonesia. 1998. p. 17 22.

6. Mason JD, Eley BM. Outline of Periodontics. $3^{\text {rd }}$ ed. Oxford: Wright. 1995. p. 114-122.

7. Murphy J, Solomon J, Ratcliffe M, Heslop N, Richard R. Science web reader: Chemistry. 2000

8. Imfeld T. Chewing gum-facts and fiction: A review of gum chewing and oral health. Crit Rev Oral Biol Med. 1999;10:405-19.

9. Addy M, Perriam E, Sterry A. Effects of sugared and sugar-free chewing gum on the accumulation of plaque and debris on the teeth. J Clin Periodontol. 1982 Jul;9(4):34654.

10. Carvalho JC, Ekstrand KR, Thylstrup A. Dental plaque and caries on occlusal surfaces of first permanent molars in relation to stage of 
eruption. J Dent Res. 1989 May;68(5):773-9.

11. Addy M, Renton-Harper P, Myatt G. A plaque index for occlusal surfaces and fissures. Measurement of repeatability and plaque removal. J Clin Periodontol. 1998;25(2):164-8.

12. Levinkind M, Owens J, Morea C, Lang NP, Addy $M$. Validation of a site specific occlusal plaque index to evaluate tooth cleaning by brushes and chewing gum. J Clin Period 1999;26(3): 177-87.
13. Remacle C, Reusens B. Functional foods, ageing and degenerative disease. 2004.

14. Addy M, Willis L, Moran J. Effect of toothpaste rinses compared with chlorhexidine on plaque formation during a 4-day period. J Clin Periodontol. 1983;10(1):89-99.

15. Quigley GA, Hein JW. Comparative cleansing efficiency of manual and power brushing. J Am Dent Assoc. 1962;65:26-9. 